\title{
Sound propagation over underlying surface
}

\author{
Valeriya K ulkina and Alexandr Komkin* \\ B M STU, Power Engineering Department, 105005, M oscow, Russia
}

\begin{abstract}
The features of noise emission, when its source is located above the underlying (reflecting) surface, have been considered. The main factor determining the pattern of sound pressure distribution in space is the interference between sound waves incident on this surface and reflected from it. The influence of the height of the noise source above the underlying surface, as well as the acoustic characteristics of this surface, on this process has been analyzed.
\end{abstract}

\section{Introduction}

Automobiles are the main source of acoustic pollution in the environment. Therefore, much attention is paid to research on reducing vehicle noise [1-3]. It happens very often in technical acoustics, that influence on the obtained results of the reflecting (underlying) surface is neglected during measurements or noise calculations, when the noise source is located at a certain height above the reflecting (underlying) surface. Thus, the noise emitted by the car exhaust system is measured in accordance with regulatory requirements (GOST 52231-2004) at a control point at a distance of $0.5 \mathrm{~m}$ from the cutoff of the exhaust pipe, at an angle of 450 from its axis. It is assumed that to assess the contribution of the exhaust noise to the external noise of the car as a whole, it is sufficient to recalculate it simply at a distance of $7.5 \mathrm{~m}$, namely, to subtract the correction $20 \lg (7.5 / 0.5)=23.5 \mathrm{~dB}$ from the sound pressure levels measured by this way in the near field of sound pressure levels.

However, this approach does not specify and does not take into account the fact that such noise measurements register the total sound pressure - the sum of the direct field and reflected from the underlying surface, usually asphalt. The reflected field is approximately equal in amplitude to the incident field, and the phase depends significantly on the frequency. Therefore, the total field, due to the interference, will differ significantly from the direct field, and this may affect the determination of the emitting power. The contribution of the reflected field will also be in the re-counting point, in the zone of the far field, but it will be different. Therefore, it is necessary to give a quantitative assessment of this factor and justify the need to take it into account in practice during conducting acoustic measurements.

In general statement this problem has been considered in many works [4-8]. The main attention has been paid to the description of acoustic characteristics of the underlying surface and mathematical modeling of noise propagation over such a surface. As for the question of the specifics of this influence on the measurement results, depending on the location of the

* Corresponding author: akomkin@mail.ru 
noise source and the calculation point above the underlying surface, this question has been considered to a lesser extent. This work is devoted to the consideration of this issue.

\section{Mathematical model}

For simplified calculations the radiation field structure is built in the calculated point of the space $P$, so the total field is a superposition of waves coming via the direct beam from the source $S$ and the beam reflected from the underlying surface, which is equivalent to the presence of the imaginary source $S_{l}$ (Figure 1).

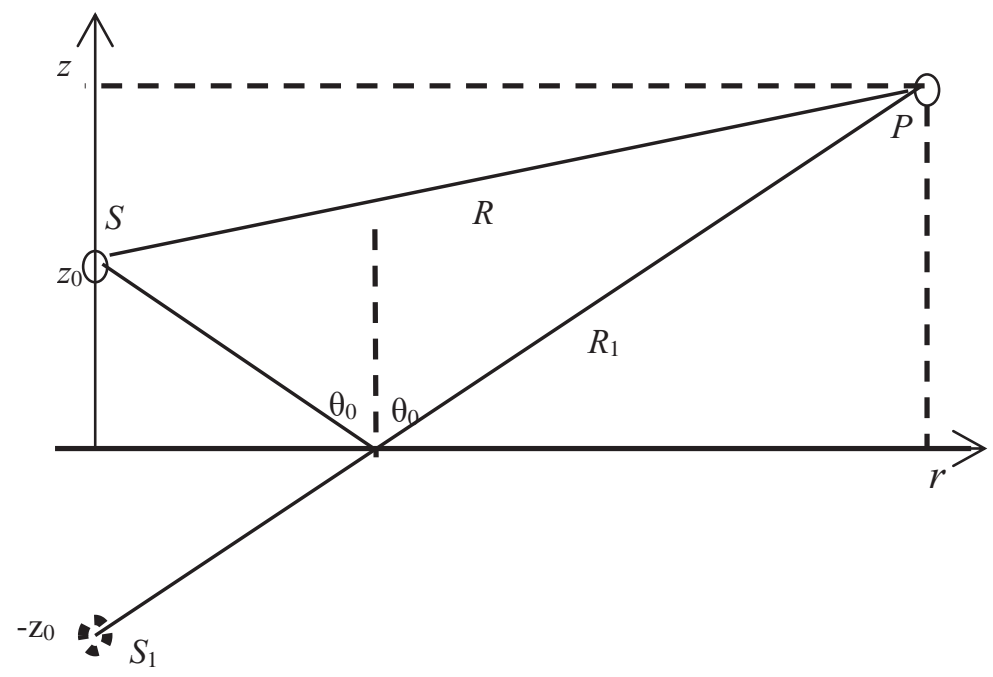

Fig. 1. Scheme of the sound propagation over underlying surface.

The underlying surface is described by an acoustic impedance $Z$, normalized to the characteristic air impedance $\rho c$. It is assumed that the acoustic impedance of the underlying surface depends on the frequency and on the surface structure (porosity, stiffness of the skeleton). The impedance does not depend on the incident angle of the sound wave.

The reflected field at the calculated point is computed by taking into account the reflection coefficient (in fact, this is an approximate solution that is suitable for relatively high frequencies and for incident angles that are far from sliding angles). In this case, the coefficient of reflection depends on the incident angle $\theta$, even in the case of a constant impedance $Z$ of the underlying surface. This dependence is described by the formula [9]

$$
V(\theta)=\frac{-1+Z \cos \theta}{1+Z \cos \theta}
$$

The second terms in the numerator and denominator (1) for an absolutely rigid boundary at any angles of incidence $\theta$ are much larger than the first terms, so for a rigid boundary $V=1$ . For an absolutely soft border $Z=0$, the reflection coefficient is equal to $V=-1$. These two limiting cases give a reflection coefficient, which is independent to the incident angle. Any other value of the impedance (not zero or infinity) gives a coefficient of reflection, which varies depending on an angle. Compacted soil and, especially, asphalt has large impedances with a module much larger than unity. However, when the incident angle $\theta$ approaches to $\pi / 2$, i.e. at sliding angles, the reflection coefficient tends to -1 . 
According to the reflection theory, the full field created by a point source is written as [5]:

$$
p\left(r, z, z_{0}\right)=\frac{1}{R} \exp (i k R)+V(\theta) \frac{1}{R_{1}} \exp \left(i k R_{1}\right),
$$

where $R=\left[\left(z-z_{0}\right)^{2}+r^{2}\right]^{1 / 2} ; R_{1}=\left[\left(z+z_{0}\right)^{2}+r^{2}\right]^{1 / 2}$, and the incident angle of the reflected beam is determined by the formula:

$$
\cos \theta=\frac{z+z_{0}}{\sqrt{\left(z+z_{0}\right)^{2}+r^{2}}} .
$$

To estimate the influence of the underlying surface on the measurement results, we introduce an auxiliary value based on the formula (2)

$$
d p_{r}=1+V(\theta) \frac{R}{R_{1}} \exp \left[i k\left(R_{1}-R\right)\right]
$$

showing the degree of change in the sound pressure at the calculation point due to the presence of the underlying surface.

The reflection coefficient included in (2) is calculated by the formula (1), and the frequency-dependent impedance $Z$ is determined on the basis of experimental studies at the normal incidence of the sound wave [4] or by calculation.

There is a large number of models for estimating the acoustic impedance of the underlying surface [8]. When developing models, the attention is paid to their simplicity - a small number of free parameters should describe the experiment in the widest possible range of frequencies.

One of the simplest ways to describe the underlying surface is to use the phenomenological models that use directly experimental data obtained in the impedance tube on cut-out samples of road surface, or in full-scale measurements. One of these models for acoustic impedance $Z$, given in [6], has the following form:

$$
Z=\left[\rho_{g}(\omega) K_{g}(\omega)\right]^{1 / 2} \Omega^{-1}
$$

where $\rho_{g}(\omega)=\rho_{0} q^{2}\left(1+i f_{\mu} / f\right)$ is the effective density, $K_{g}(\omega)=\gamma P_{0}\left[1+(1-\gamma) /\left(1-i f / f_{\theta}\right)\right]^{-1}$ is the effective elasticity, $f_{\mu}=\Omega r_{s} /\left(2 \pi \rho_{0} q^{2}\right), f_{\theta}=r_{s} /\left(2 \pi \rho_{0} N_{p r}\right), \Omega$ is the porosity, $q^{2}$ is the shape parameter (convolution), $P_{0}$ is the atmospheric pressure, $\gamma$ is the ratio of heat capacity, equal to 1.4 for air; $P_{\mathrm{r}}$ is the Prandtl number - ratio of kinematic viscosity coefficient to the thermal conductivity coefficient, $r_{s}$ is the coefficient of flow resistance with a dimension of $\mathrm{N} \cdot \mathrm{s} / \mathrm{m}^{3}$.

Meanwhile, the semiempirical Delaney-Bazley formula, proposed in the early 70 s of the last century, still finds the greatest use [10]. In this case, the normalized acoustic impedance of the sound-absorbing material is determined by the following simple formula

$$
z_{p}=1+9,08\left(f / \sigma_{p}\right)^{-0,75}+j 11,9\left(f / \sigma_{p}\right)^{-0,73}
$$

where $\sigma_{p}$ is the flow resistance of sound-absorbing material, $\mathrm{kPa} \cdot \mathrm{s} \cdot \mathrm{m}^{-2}$. 
According to the work [7] this value varies for asphalt from $5 \mathrm{MPa} \cdot \mathrm{s} \cdot \mathrm{m}^{-2}$ for "fresh" asphalt to $30 \mathrm{MPa} \cdot \mathrm{s} \cdot \mathrm{m}^{-2}$ for "old" asphalt.

\section{The emission of an automobile exhaust system}

Let's consider the application of this model for the emission simulation of an automobile exhaust system at the calculated points.

In relation to automobile specifics, this problem has first been formulated in [11]. In this work, not only an analytical model of the underlying surface has been considered on the base of the results of noise estimation at the calculation point, but the research in finite element modeling of this problem had been carried out and the results of numerical calculations were presented. Let's examine this issue in details.

The following case will be considered. The exhaust pipe is located at a height of $\mathrm{z}_{0}=0.3 \mathrm{~m}$ from the underlying surface. Graphs of the $d_{p r}$ function for a point located in the near field at a standard distance of $r=0.5 \mathrm{~m}$ from the pipe section at a height $z=0.3 \mathrm{~m}$, for two values of the blowing resistance $\sigma p$ are shown in Figure 2, a.
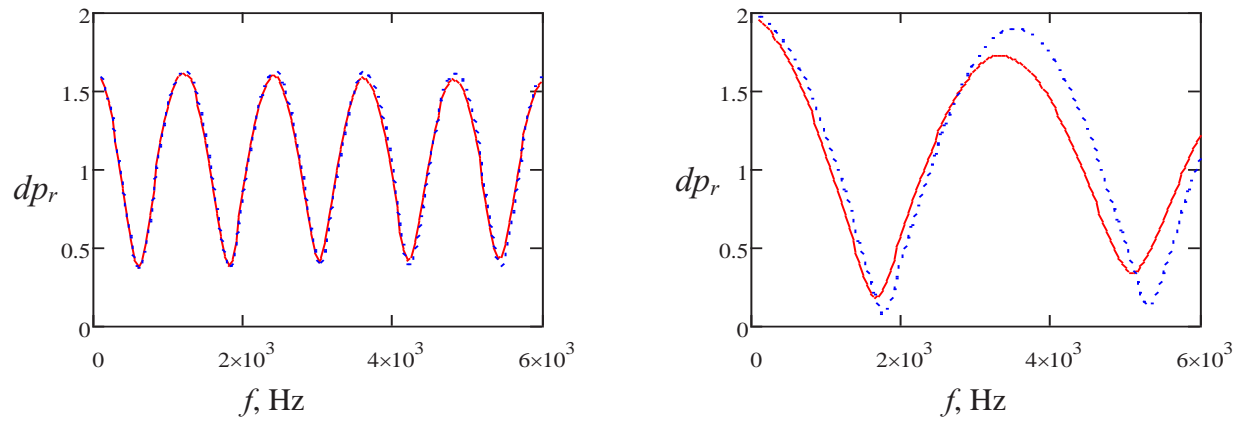

Fig. 2. Dependence of the $d p_{r}$ on the frequency in the near field (a) and far field (b) for: $(-) \sigma p=5 \mathrm{MPa} \cdot \mathrm{s} \cdot \mathrm{m}-2$ and $(---) \sigma \mathrm{p}=30 \mathrm{MPa} \cdot \mathrm{s} \cdot \mathrm{m}-2$.

Graphs of the $d_{p r}$ function for a point located in the far field and corresponding to the measurement of an automobile external noise, with coordinates $r=7.5 \mathrm{~m}$ and $z=1.2 \mathrm{~m}$, for the same values of the blowing resistance op are shown in Figure 2, b.

As follows from the presented graphs, the influence of the underlying surface on the results of noise measurements leads to the fact that the noise spectrum at the calculated point is modulated in frequency. Moreover, the closer the calculated point is to the noise source, the higher the frequency of modulation is. At long distances, there is also a decrease in the amplitude of modulation with frequency in addition to reducing the frequency of modulation.

Also, the presented results show that the blowing resistance has a noticeable effect on the measurement results only at large distances from the source, and this effect increases with frequency. The effect of this parameter is insignificant at measuring in the near field.

It follows from the presented results, that the conversion of noise levels from distance $\mathrm{r} 1$ to distance $r_{2}$ in the presence of the underlying surface will be correct if, in addition to the traditional correction for the distance $\Delta L_{r}=20 \lg \left(r_{2} / r_{1}\right)$, a correction for the underlying surface is also introduced, determined by the ratio

$$
\Delta L_{\text {п }}=20 \lg \left(d p_{r 2} / d p_{r 1}\right)
$$

The graph of the correction $\Delta L_{n}$ calculated by the formula (6) for the two considered calculated points is shown in Figure 3. 
It follows from the figure 3 that the value of the correction $\Delta L_{\Pi}$ has significant frequency fluctuations with an amplitude exceeding $10 \mathrm{~dB}$. So, its accounting is necessary for recalculating the output noise measured in the near field to the far field. Otherwise, the error will reach a significant amount with this recalculation.

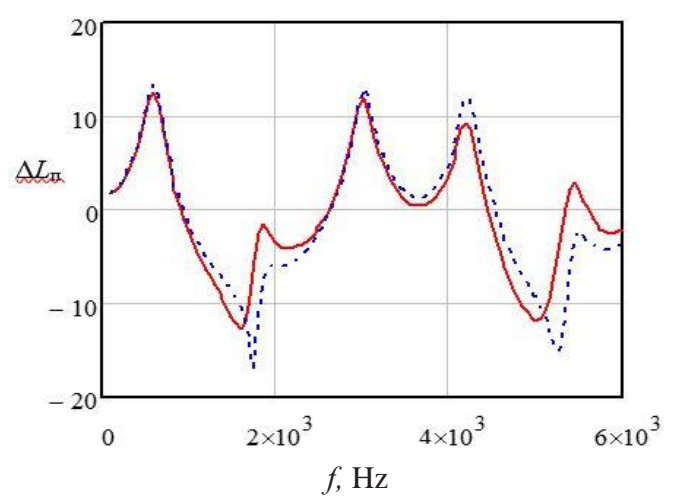

Fig. 3. Dependence of the $\Delta L_{\Pi}$ value on the frequency for: $(-) \sigma_{\Pi}=5 \mathrm{MPa} \cdot \mathrm{s} \cdot \mathrm{m}^{-2}$ and $(---) \sigma_{\Pi}=30 \mathrm{MPa} \cdot \mathrm{s} \cdot \mathrm{m}^{-2}$.

\section{Acoustic screen}

The design scheme of an acoustic screen on the underlying surface is shown in Figure 4. The rays here show how direct sound from the source gets first to the upper edge of the screen, and then after diffraction - to the calculation point. It also shows the pattern of reflected rays forming both in the area in front of the screen and behind it.

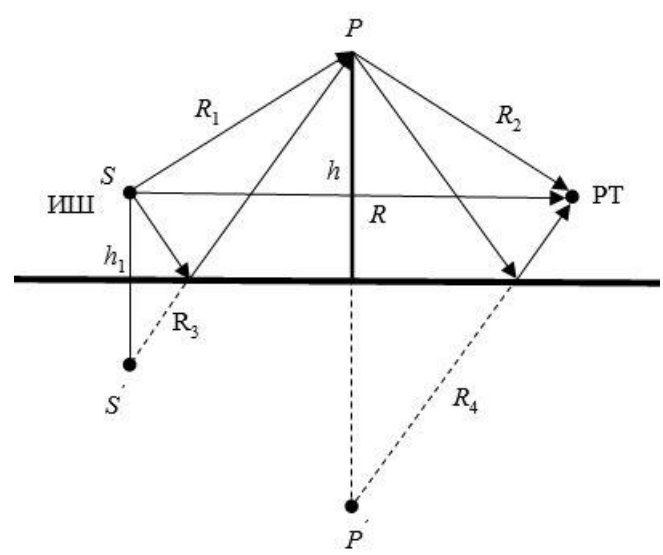

Fig. 4. Calculation scheme for determining an acoustic efficiency of the screen on the underlying surface.

If there is no underlying surface, the acoustic efficiency of the screen $\Delta L, \mathrm{~dB}$, will depend on the Fresnel number $N=2 \delta / \lambda$, where $\delta$ is the path difference, $\delta=R_{1}+R_{2}-R$; and $\lambda$ is the sound wave length. For $N \geq 1$ this value is determined by the Maekawa's formula:

$$
\Delta L=10 \lg (20 N) .
$$


If there is an underlying surface, the regularities of changing the acoustic efficiency of the screen $\Delta \mathrm{L}$ become much more complex. In this case, the estimation of $\Delta \mathrm{L}$ is no longer reduced to determining the Fresnel number, but depends on a larger number of factors. The main determining factor in this case is the interference between the incident and reflected waves in the space both in front of the screen and behind it. We'll focus on the issue of interference of sound waves in the considered system in details.

We'll consider the case when sound reflection occurs only in front of the screen. As follows from Figure 4 the interference in the space in front of the screen can be described using the model which is discussed above. In this case, we introduce an auxiliary value by analogy (4) to estimate the influence of the underlying surface:

$$
d p_{13}=1+V(\theta) \frac{R_{1}}{R_{3}} \exp \left[i k\left(R_{3}-R_{1}\right)\right]
$$

Graphs of the $d p_{13}$ function for the case when the noise source and the calculation point are located at a height of $1 \mathrm{~m}$ above the underlying surface for two values of its blowing resistance $\sigma$. It follows from the presented graphs, that changes in the blowing resistance within the considered limits (by more than an order of magnitude) lead to weak changes in the $d p_{13}$ values, except for small $\mathrm{h}$, when they become more noticeable. It should be noted that for $\sigma=30 \mathrm{MPa} \cdot \mathrm{s} \cdot \mathrm{m}^{-2}$, the $d p_{13}$ values are almost equal to those calculated for an absolutely rigid surface.

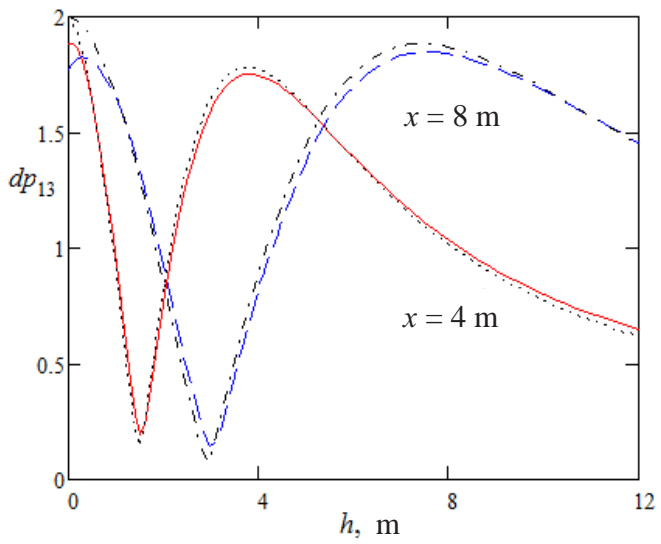

(a)

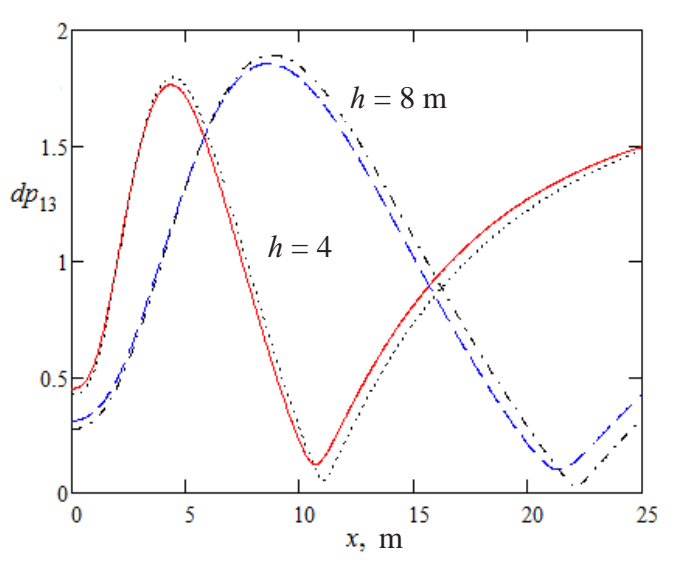

(b)

Fig. 5. Dependence of $d p_{13}$ on the screen height $h(\mathrm{a})$ and the distance $x(\mathrm{~b})$ for frequency $250 \mathrm{~Hz}$ with: $(-$ and --$) \sigma_{\Pi}=1 \mathrm{MPa} \cdot \mathrm{s} \cdot \mathrm{m}^{-2}$ and $(\cdots \cdots$ and $\cdots \cdot \cdot) \sigma_{\Pi}=30 \mathrm{MPa} \cdot \mathrm{s} \cdot \mathrm{m}^{-2}$.

The dependencies of the $d p_{13}$ function on the screen height $h$ show (Figure 5, a) that this function changes significantly with the growth of $h$. For $h \rightarrow 0$, the $d p_{13}$ function takes a maximum value equal to 2 , regardless to the distance $x$, i.e. the pressure becomes equal to the pressure of the incident wave multiplied by two. In all other cases, the behavior of $d p_{13}$ largely depends on the distance from the source to the screen. The graphic dependencies in this figure have a strongly marked minimum, and the higher the $x$ value is, the higher is the screen height of this minimum. And in general, it can be noted that the increase in $x$ leads to the" stretching " of the $d p_{13}(h)$ graphic dependence in the horizontal direction. Figure 10 presents comparison of efficiencies of a screen on the ground and a semi-infinite screen with the same $h_{e}=3 \mathrm{~m}$, which shows that a decrease in the $R_{I}$ reflection coefficient of the ground, 
as expected, increases the efficiency of a screen on the ground. At the same time, comparison of the results in Figure 9 and Figure 10 demonstrates that, the decrease in screen efficiency due to presence of the ground does not depend on the effective screen height.

The dependences of the dp13 function on the position of the noise source $x$ (Figure 5,b) show that this function also undergoes significant changes. It has a local minimum, the position of which depends on the screen height. So that a higher height $\mathrm{h}$ corresponds to a higher $\mathrm{x}$ value. A distinctive feature of the $d p_{13}(\mathrm{x})$ dependence is that it does not tend to the maximum for $x \rightarrow 0$. Its value depends on the screen height here, so the larger the $h$ is, the smaller is the function value.

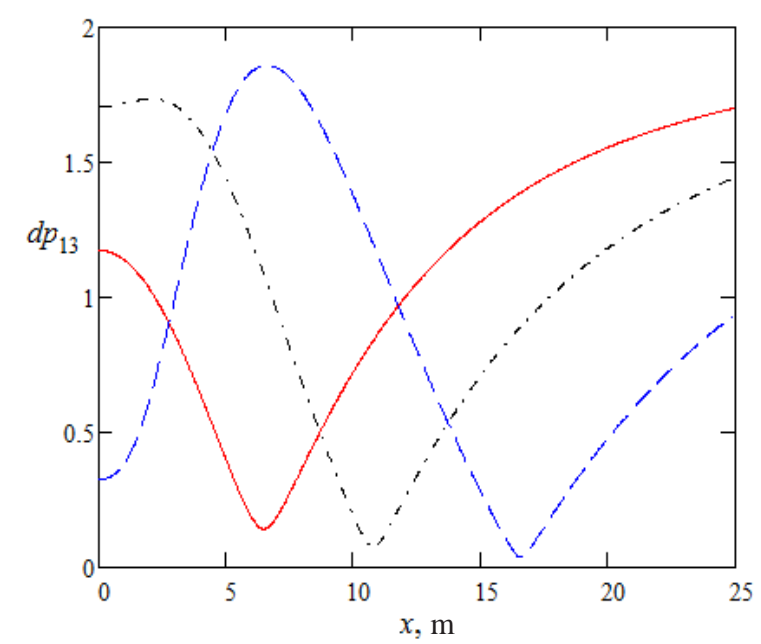

Fig. 6. Dependence of $d p_{13}$ on the distance $x$ for frequencies:

$(-) 125 \mathrm{~Hz},(-\cdots) 175 \mathrm{~Hz}$ and $(--) 250 \mathrm{~Hz}$

The dependences of the $\mathrm{dp}_{13}$ function on the distance $\mathrm{x}$ of the noise source to the screen with a height of $6 \mathrm{~m}$ for sound waves of different frequencies are presented in Figure 6. As it follows from the presented graphs, the higher the sound frequency is, the greater $x$ value corresponds to the local minimum of the $d p_{13}(x)$ function. As for the value of this function at $x \rightarrow 0$, it depends on the sound frequency, or rather on the wavelength, namely, how this wavelength relates to the height of the screen and the height of the location of the sound source.

Similar results can be obtained during consideration of the sound waves interference in the space behind the acoustic screen.

It should be noted that the local minima of the $\mathrm{dp}_{13}$ function should lead to a significant increase in the acoustic efficiency of the screen. This is shown by the results of corresponding numerical calculations in [12], where it is found that when the underlying surface exists, the efficiency of the acoustic screen ceases to be a monotonously increasing function of the screen height, but changes in a much more complex way.

\section{Conclusion}

The conducted research has shown that when a sound source is located above the underlying surface, the characteristics of this source sound emission change significantly. This is shown in the examples of analyzing noise emission on a section of a car exhaust pipe and considering the acoustic efficiency of a screen located on the underlying surface. Therefore, the it is absolutely necessary to take the reflection processes on the underlying surface into consideration. 


\section{References}

1. N. D. Chaynov, V. A. M arkov, A. A., Savastenko, Structural Noise and Acoustic Characteristics Improvement of Transport Power Plants, in IOP Conference Series: $M$ aterials Science and Engineering, 327 (2), 022069. (2018)

2. V.V.Tupov, The calculation of channel end hole reflection ratio without a flange, in AIP Conference Proceedings, 2195 (1), 020034. (2019)

3. R. I. Rakhmatov, V. E. K rutolapov, V. N. Zuzov, V ibroengin. Proc. 25, 135 (2019)

4. T.F.W. Embleton, J.E. Piercy, N. Olson, J. A coust. Soc. A m. 59 (1), 267 (1976)

5. M. R. Stinson, J. A coust. Soc. A m. 98 (3),1810 (1995)

6. M.C. B erengier, M.R. Stinson, G.A. Daigle, J. A coust. Soc. A m. 101(1), 155 (1997)

7. S. S. Jung, C-H. Hwang, J ournal of the Korean Physical Society, 44(4), 868-874. (2004)

8. K. Attenborough, I. B ashir, S. Taherzadeh, J. A coust. Soc. A m. 129 (5), 2806 (2011)

9. M. A . Isakovich, General acoustics (Nauka, M oscow, 1973) [in Russsian]

10. M.E. Delany, E.N. Bazley, A ppl. A coust. 3 (1), 105 (1970)

11. A. I. Komkin, M. A. M ironov, S. I. Y udin, Simulation of acoustic radiation of the vehicle exhaust system over the underlying surface, in Proceedings of the scientific conference to the 100th anniversary of A.V. Rimsky-K orsakov, 10-11 november 2010, M oscow, Russia (2010) [in Russsian] 